\title{
Differential gene expression and co-regulated expression of genes in leukemia: an in-silico approach to identify potent biomarker
}

\author{
D. M. Agase* \\ Department of Zoology, Govt. J.S.T.P.G. College, Balaghat (M.P.), India \\ K. K. Gupta \\ Centre for Bioinformatics, Pondicherry University, Puducherry, India
}

A. Wasnik

Department of Botany, Govt. J.S.T.P.G. College, Balaghat (M.P.), India

M. S. Markam

Department of Zoology, Govt. J.S.T.P.G. College, Balaghat (M.P.), India

S. B. Zade

Department of Zoology, R.T.M. Nagpur University, Nagpur (M.S.), India

P. M. Mohurle

Department of Zoology, R.T.M. Nagpur University, Nagpur (M.S.), India

T. S. Kothe

Department of Zoology, Govt. K.N.G. College, Balaghat (M.P.), India

*Corresponding author. Email: sbt.durgesh@gmail.com

\section{Article Info}

https://doi.org/10.31018/

jans.v13i2.2650

Received: March 28, 2021

Revised: May 22, 2021

Accepted: May 26, 2021

\section{How to Cite}

Agase, D.M. et al. (2021). Differential gene expression and co-regulated expression of genes in leukemia: an in-silico approach to identify potent biomarker. Journal of Applied and Natural Science, 13(2), 585 - 592. https://doi.org/10.31018/jans.v13i2.2650

\begin{abstract}
A biomarker can be measured, used to diagnose or classify disease, and measure progress as well as the therapeutic response of the disease. Early diagnosis and selection of appropriate treatment can be critical for the successful treatment of diseases. Identification and characterization of potent diagnostic biomarkers, and therapeutic targets rely heavily on traditional in vitro screens which require extensive resources and time. Integration of in silico screens prior to experimental validation can improve the efficiency and potency of biomarkers as well as reduce the cost and time of biomarker discovery. Considering the need, present work was undertaken to identify biomarkers for different classes of leukemia. Differential Gene Expression (DGE) analysis and co-regulated expression analysis were used for in silico identification and characterise a potent biomarker for leukemia. On the basis of in silico screening, the present study proposed seven protein-coding (CD38, TSC22D3, TNFRSF25, AGL, LARGE1, ARHGAP32, and PARM1) genes for the diagnosis of leukemia. The study also proposed a novel three-step lineagespecific model for the diagnosis of leukemia. In the three-step diagnosis model, the first group of biomarkers with an association of clinical and hematological parameters diagnose leukemia. The second group of biomarkers diagnoses acute and chronic form of leukemia. The third group of biomarkers identifies whether it belongs to myeloid lineage or lymphoid lineage.
\end{abstract}

Keywords: Biomarkers, Co-regulated Expression, Differential Gene Expression, Leukemia

\section{INTRODUCTION}

Leukaemia is a malignant neoplasm of hematopoietic cells. Aberrant gene expression related to cellular differentiation, and proliferation of hematopoietic cell contributes to malignant transformation of cells. A better understanding of differential gene expression (DGE) in leukemia should provide diagnostic biomarkers, and therapeutic targets for therapy of this disease. Differential gene expression analysis refers to the analysis, and interpretation of differences in the abundance of gene transcripts within a transcriptome (Conesa et al., 2016). DGE is important to understand the biological differences between healthy and diseased states (Rodriguez et al., 2017). Identification and characterization of potent diagnostic biomarkers, and therapeutic targets rely heavily on traditional in vitro screens which require extensive resources and time. Integration of in silico screens prior to experimental validation can improve the efficiency and potency of biomarkers as well as reduce the cost and time of biomarker discovery. Considering the need, present work was undertaken to 
identify biomarkers for different classes of leukemia. In the present work, the combination of differential gene expression analysis and co-regulated expression analysis were used for in silico identification of potent biomarkers for leukemia.

\section{MATERIALS AND METHODS}

\section{Microarray datasets selection}

In order to find proper gene expression profiles in microarray datasets, a systematic search was performed in Gene Expression Omnibus (GEO) database (https:// www.ncbi.nlm.nih.gov/geo/) using the keywords "AML" and "Serum", "CML" and "Serum", "Acute Lymphoblastic Leukemia" and "Serum". These broad searches yielded a large number of gene expression data sets. The search was first narrowed down to a few potential data sets using various filters such as "Homo sapiens" and "Expression Profile by Array" in the GEO database. Finally, by detailed study of data sets four normalized data sets were selected. The flowchart of the dataset selection procedure, filter features of data sets, and information of datasets are shown in Fig. 1.

\section{Selection and combination of top differentially} expressed gene (DEG)

Differentially expressed genes of four different types of leukemia were analyzed using the online tool GEO2. GEO2R is an interactive tool that enables the analysis of GEO datasets. It uses a web-based program, that employs the Bioconductor packages, and limma $R$ (Gentleman et al., 2004; Smyth, 2004). A Venn diagram was prepared for the combination of four different sets of four different types of leukemia. InteractiVeen (Heberle et al., 2015) online tool was used to combine all data set to find out overlapping and non-overlapping differentially expressed gene. In order to find the best common biomarker for all classes of leukemia, and to prevent missing critical genes, it was decided to select differentially expressed gene overlapped between at least two data sets.

\section{Area under curve (AUC) analysis}

The expression values of selected overlapping differentially expressed genes were extracted in the form of a soft file, and imported to GraphPad Prism software. Receiver-operating characteristic (ROC) curves, and area under the ROC curve (AUC) were used to assess the detection ability of each differentially expressed gene in the diagnosis of different classes of leukaemia from the control group based on the sensitivity and specificity of each differentially expressed gene. The higher the AUC, better the model would be.

\section{Hierarchical clustering analysis}

Individual expression values of significantly up/down-

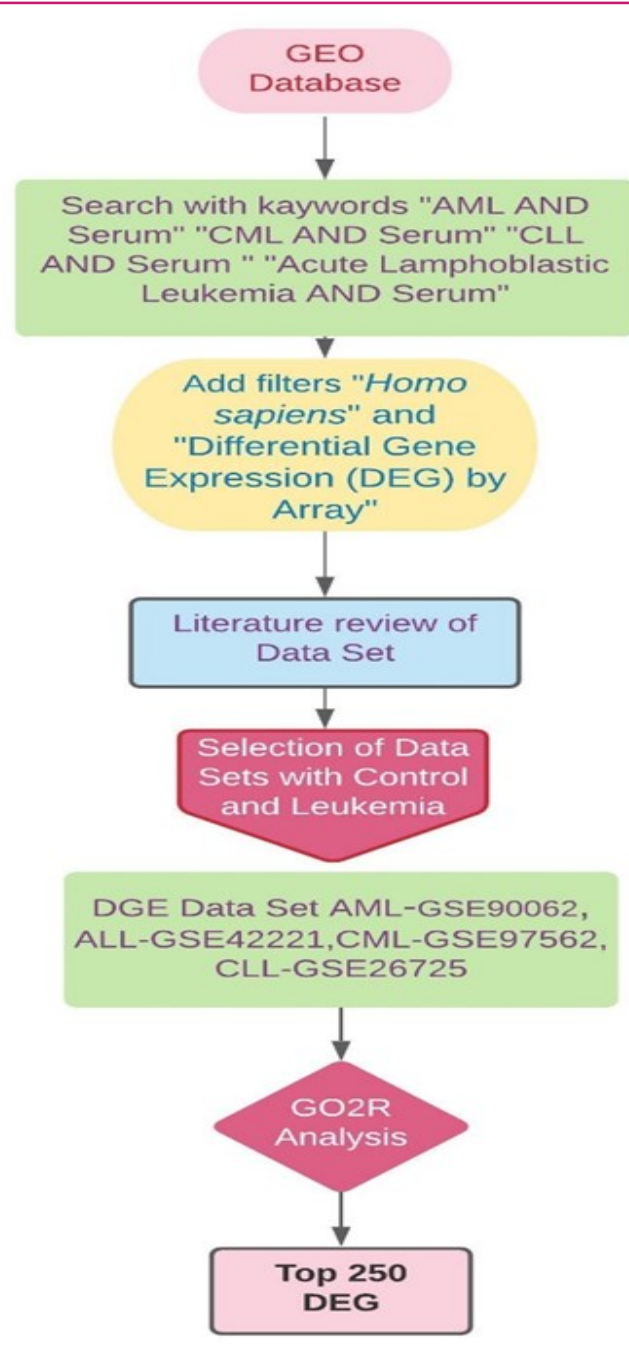

Fig. 1. Microarray dataset selection procedure for $G O 2 R$ Analysis, and identification of top 250 differentially expressed genes for four classes of leukemia.

regulated differentially expressed genes in leukaemia were logarithm transformed, and were used as input values for the hierarchical clustering algorithm. The following criteria were applied: the distance chose "Pearson Correlation", and the linkage selected "average". The result is demonstrated as a Heatmap. Heatmapper webserver was used for the preparation of the heatmap.

\section{RESULTS}

\section{Microarray dataset selection}

The selected microarray dataset and their features for different classes of leukemia are shown in Table 1. The volcano plot and mean difference plot are presented from Fig. 2- 9 for each selected microarray dataset. A volcano plot displayed statistical significance $(-\log 10 \mathrm{P}$ value) versus magnitude of change (log2 fold chain) of differentially expressed gene and the mean difference plot displays log2 fold chain versus average log2 expression value. Highlighted genes were significantly 
differentially expressed at a default adjusted $p$-value cut-off of 0.05 (red= upregulated and blue= downregulated).

\section{Selection and combination of top differentially ex-} pressed gene (DEG)

After GEO2R analysis, 164 genes for AML, 202 genes for ALL, 146 genes for CLL and 194 for CML showed significantly up/downregulation. After integration of the results with the help of Venn diagram, one gene was common in AML, ALL and CLL, six genes were common in Acute leukemias (ALL and AML), five genes were common in Chronic leukemias (CML and CLL), four genes were common in Myeloid leukemias (AML and $\mathrm{CML}$ ) and six genes were common in Lymphoid leukemias (ALL and CLL). Details of common genes are shown in Fig. 10 and Table 2.

\section{Area under curve (AUC) analysis}

On the basis of the lowest adjusted $p$ value of common differentially expressed seven genes from different groups (CD38, TSC22D3, TNFRSF25, AGL, LARGE1, ARHGHP32, and PARM1) were selected for further analysis. After extraction of expression values for all seven DEGs, in order to find the reliable marker in discriminating leukemia from healthy controls, AUC analysis was performed and ROC curves for seven DEG were prepared. ROC curve AUC values of seven DEG for different groups are shown in Table 3.

\section{Hierarchical clustering analysis and co-expression correlation}

Fig. 11-14 demonstrates hierarchically clustered Heatmaps built up using the expression values of
DEGs for four classes of leukaemia. Four differentially expressed genes AGL, CD38, TNFRSF25, TSC22D3 are shown in the Heatmap of AML. AGL and CD38 showed downregulation and TNFRSF25, TSC22D3 showed upregulation in AML patients. Similarly, four differentially expressed genes LARGE1, TNFRSF25, CD38, TSC22D3 are shown in the Heatmap of ALL. LARGE1, TNFRSF25, and TSC22D3 showed coexpression and upregulation in ALL patients and CD38 showed down-regulation. Three differentially expressed genes AGL, ARHGAP32, and PARM1 are shown in the Heatmap of CML. ARHGAP32 and PARM1 showed downregulation and $A G L$ showed upregulation in $C M L$ patients. Four differentially expressed genes LARGE1, ARHGAP32, CD38, and PARM1 are shown in the Heatmap of CLL. All four genes showed similar coexpression patterns and down-regulated in CLL.

\section{DISCUSSION}

In recent years, there has been an increased emphasis on personalized and targeted therapy for leukemia patients. Therefore, it is important to explore the mechanism for the development of novel therapeutic strategies. Recently, differential gene expression profiling analysis has been widely used to reveal abnormal gene expression patterns related to leukemia, to identifying novel diagnostic and therapeutic targets. In the present study, differential gene expression analysis and co-regulated gene expression analysis were used to identify some novel diagnostic and therapeutic targets.

By using in silico methods, the present study proposed seven new protein-coding genes for the diagnosis of

Table 1. Selected microarray dataset for GO2R analysis, and identification of top 250 differentially expressed genes for four classes of leukemia.

\begin{tabular}{llllll}
\hline S.N. & GEO accession number & Leukemia & Platform & Samples & Authors \\
\hline 01 & GSE90062 & AML & GPL15207 & Control=03, AML=03 & Li et al., 2017 \\
02 & GSE42221 & ALL & GPL96 & Control=04, ALL=07 & Harder et al., 2013 \\
03 & GSE97562 & CML & GPL6244 & Control=20, CML=20 & Aviles et al., 2017 \\
04 & GSE26725 & CLL & GPL570 & Control=05, CLL=12 & Vargova et al., 2011 \\
\hline
\end{tabular}

Table 2. Details of Common Differentially Expressed Gene (DEG).

\begin{tabular}{lll}
\hline Leukemia & $\begin{array}{l}\text { Number of common } \\
\text { protein coding DEG }\end{array}$ & Symbol of common protein coding DEG \\
\hline AML, ALL, CLL & 1 & CD38 \\
ALL, AML & 6 & GJA1, SH3BP5, SLC1A4, TSC22D3, PHGDH, TNFRSF25 \\
CLL, CML & 5 & ARHGAP32, FRY, CAMK1D PIK3R6, PARM1 \\
AML, CML & 4 & AGL, LHFP, CHCHD7, TMEM154 \\
ALL, CLL & 6 & ANP32E, LARGE1, IGLC1, CYAT1, SMAGP, ITM2C \\
\hline
\end{tabular}


Table 3. AUC analysis for seven DEGs.

\begin{tabular}{lllll}
\hline Leukemia type & Gene & Area & Stander error & p value \\
\hline AML & CD38 & 0.8762 & 0.0524 & 0.0001 \\
AML & TSC22D3 & 1.0000 & 0.0000 & 0.0495 \\
AML & TNFRSF25 & 1.0000 & 0.0000 & 0.0495 \\
AML & AGL & 1.0000 & 0.0000 & 0.0490 \\
ALL & CD38 & 1.0000 & 0.0000 & 0.0082 \\
ALL & TSC22D3 & 1.0000 & 0.0000 & 0.0082 \\
ALL & TNFRSF25 & 1.0000 & 0.0000 & 0.0495 \\
ALL & LARGE1 & 1.0000 & 0.0082 & 0.0082 \\
CML & ARHGAP32 & 0.8800 & 0.0590 & 0.0001 \\
CML & PARM1 & 0.8825 & 0.0549 & 0.0001 \\
CML & AGL & 0.08513 & 0.0598 & 0.0001 \\
CLL & CD38 & 1.0000 & 0.0000 & 0.0016 \\
CLL & ARHGAP32 & 0.9236 & 0.07371 & 0.0004 \\
CLL & PARM1 & 1.0000 & 0.0000 & 0.0016 \\
CLL & LARGE1 & 1.0000 & 0.0000 & 0.0016 \\
\hline
\end{tabular}

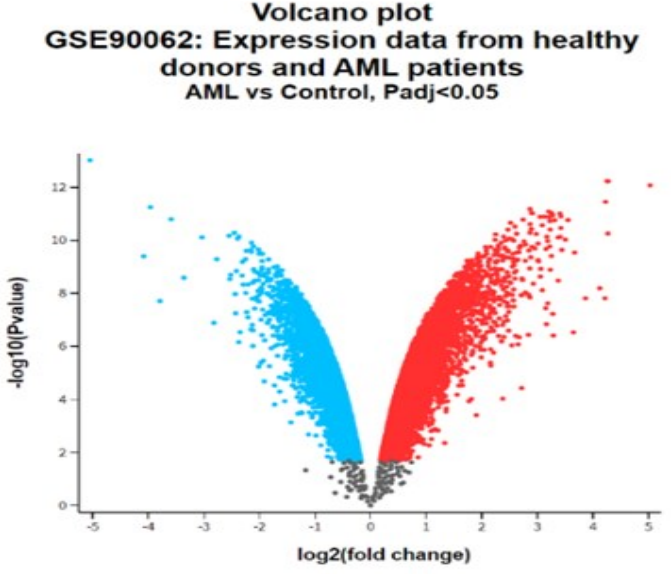

Fig. 2. Volcano plot of $A M L$.

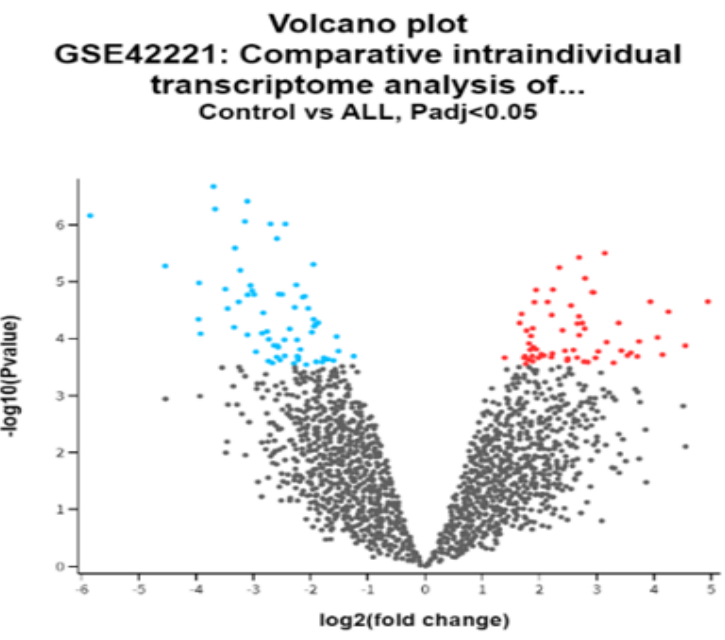

Fig. 4. Volcano plot of ALL.

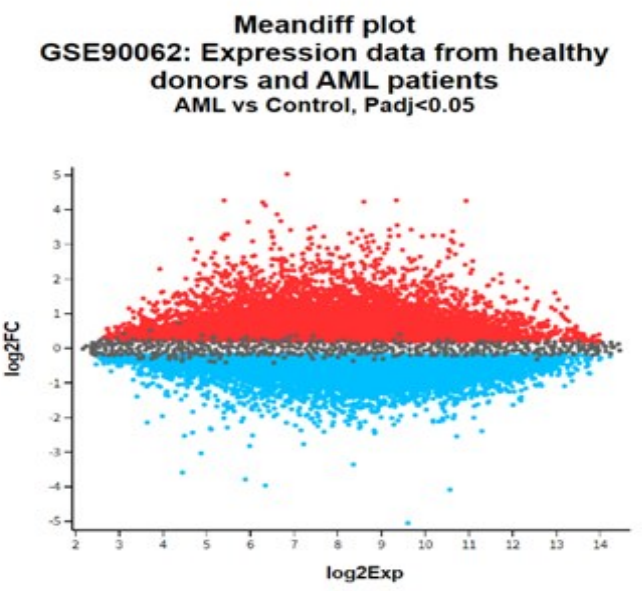

Fig. 3. Mean difference plot of $A M L$

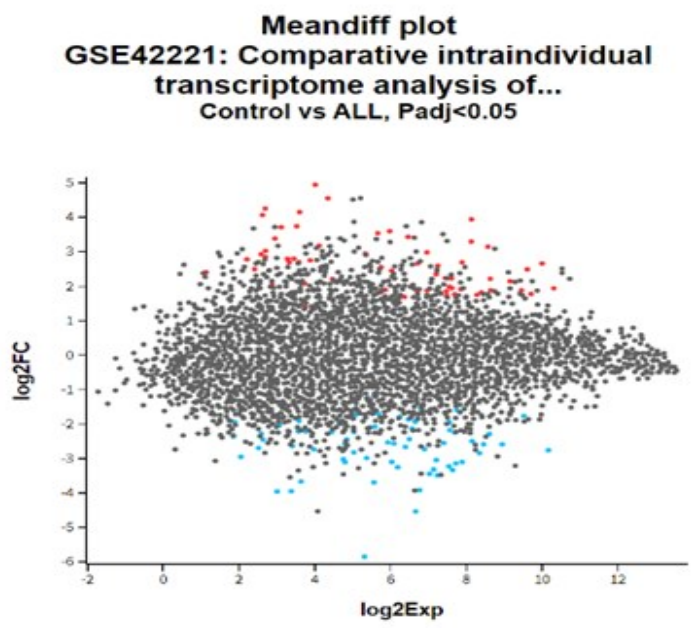

Fig. 5. Mean difference plot of ALL. 


\section{Volcano plot \\ GSE26725: Gene expression analysis of $12 \mathrm{~B}$ - cell Chronic Lymphocytic... Control vs CLL, Padj<0.05}

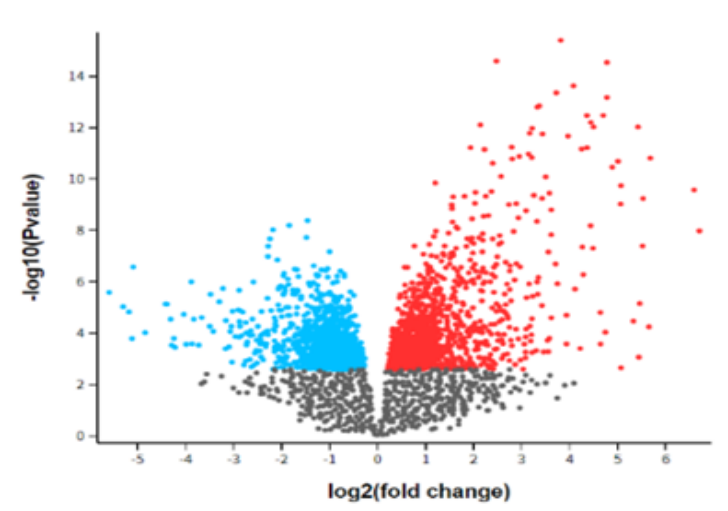

Fig. 6. Volcano plot of CLL.

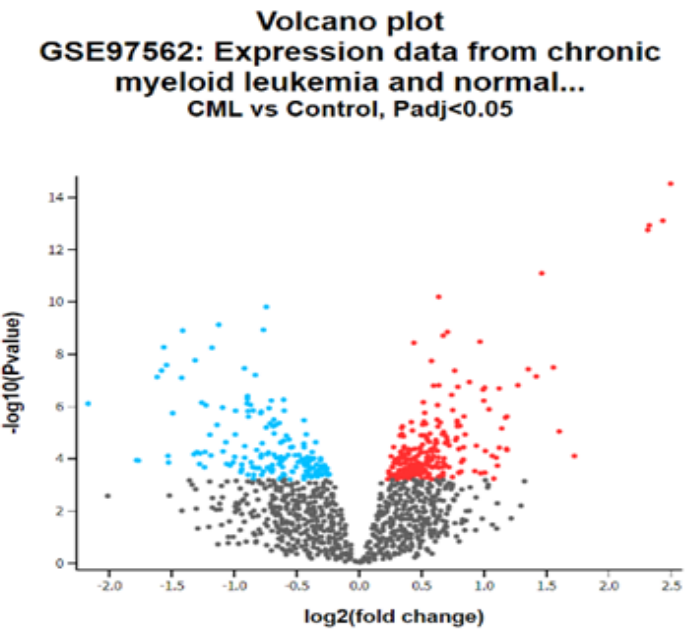

Fig. 8. Volcano plot of CML.

leukemia. To identify protein-coding genes as biomarkers, four GEO microarray datasets containing the expression profiles from the serum of leukemia patients and healthy controls were chosen for differential expression analysis. Four GEO microarray dataset GSE90062, GSE42221, GSE97562, and GSE26725 comprises gene expression data for AML, ALL, CML, and CLL respectively. The gene expression profile data were downloaded, and analyzed using the GEO2R. Top 250 genes were selected from each microarray data sets. Common genes between different classes of leukemia were captured out with the help of the Venn diagram. On the basis of the lowest adjusted $p$ values, seven genes were selected from different groups of leukemia. The expression values for all these differentially expressed genes were extracted and normalized. Using Area Under ROC curve Analysis (AUC), the most powerful DEGs in discriminating the leukemia patient from a healthy control were picked out. In the present study, a total seven genes showed AUC > 0.800 and considered for further analysis. The co-expression pat-

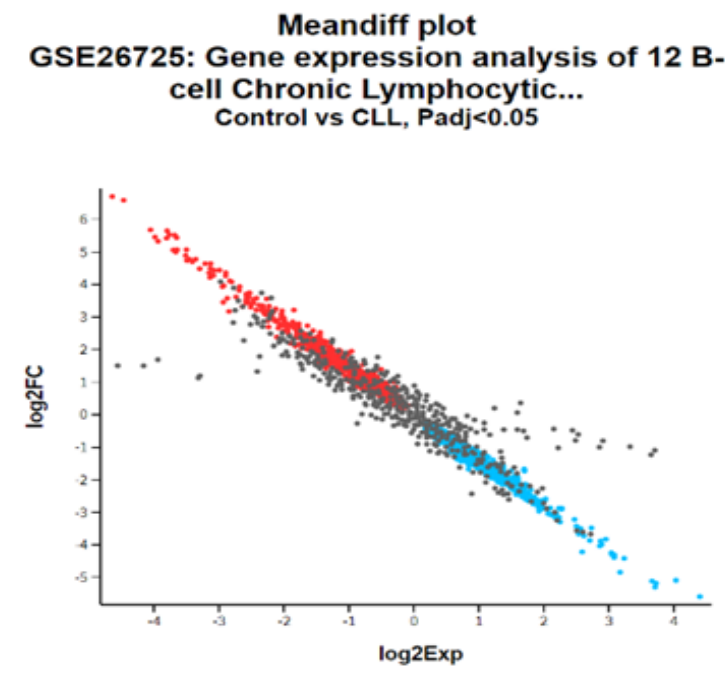

Fig. 7. Mean difference plot of CLL.
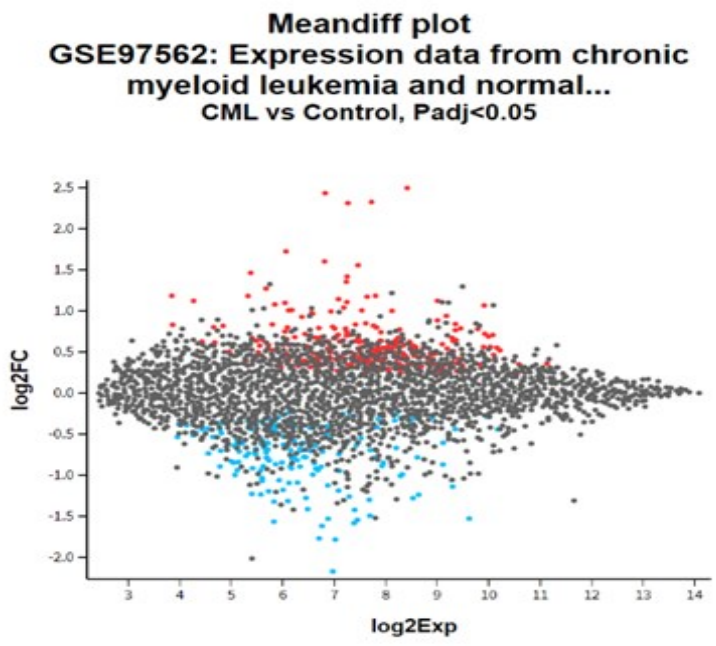

Fig. 9. Mean difference plot of CML.

tern of seven identified genes was analyzed by hierarchically clustered Heatmaps built up using the expression values of DEGs. The present study also proposed a three-step lineage-specific model for the diagnosis of leukemia. In the three-step diagnosis model, the first group of biomarkers with an association of clinical and hematological parameters diagnose leukemia, the second group of biomarkers diagnoses acute and chronic form of leukemia and the third group of biomarkers identifies whether it belongs to myeloid lineage or lymphoid lineage.

Tripathi and Pandya (2016) carried out a similar study when they studied differential analysis of gene expression for acute myeloid leukemia. They analyzed the transcriptional profiling of human T-cell involved in the AML disease and reported CD4, NA, ADTRP genes as a diagnostic and therapeutic biomarker. Tang et al. (2019) reported the five key genes: ACTR2, ARPC3, ARPC5, CTTN, and APP may be implicated in tumor progression and could potentially represent promising prognostic biomarkers and therapeutic targets for AML 


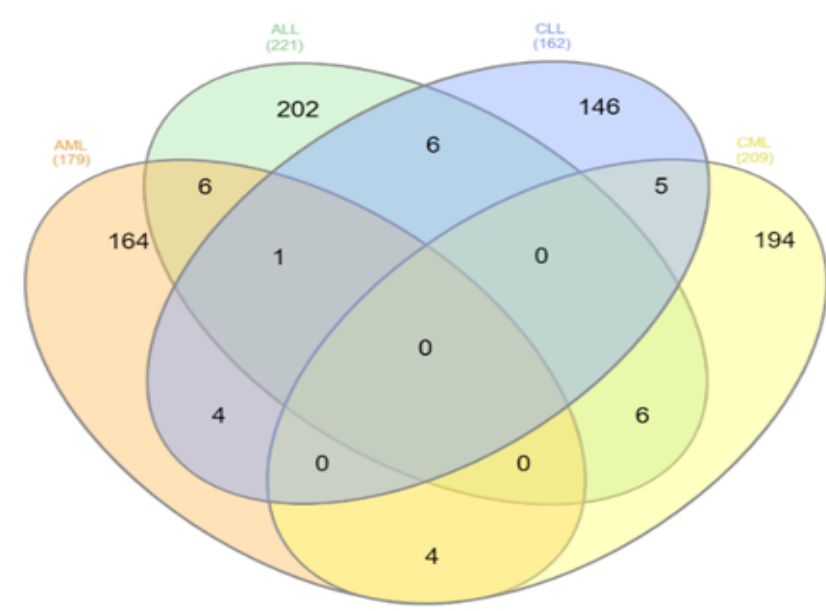

Fig. 10. Venn diagram of common differentially expressed gene (DEG).

patients. Wei Fu et al. (2020) reported abnormal expression of CYBB, CYFIP2, SERPINE1, and ITGAM based on high-throughput data analysis. Sanchez and Mackenzie (2020) reported integrative network analysis of differentially methylated and expressed genes for

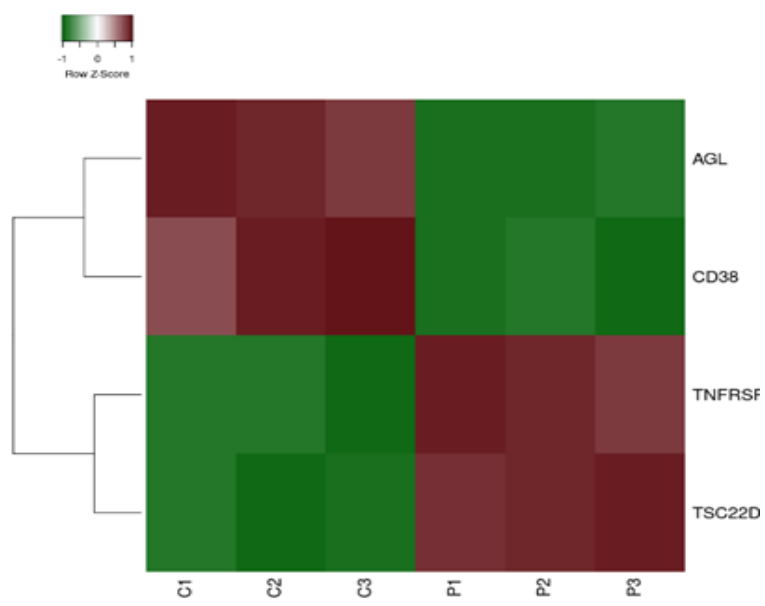

Fig. 11. Heatmap for $A M L$.

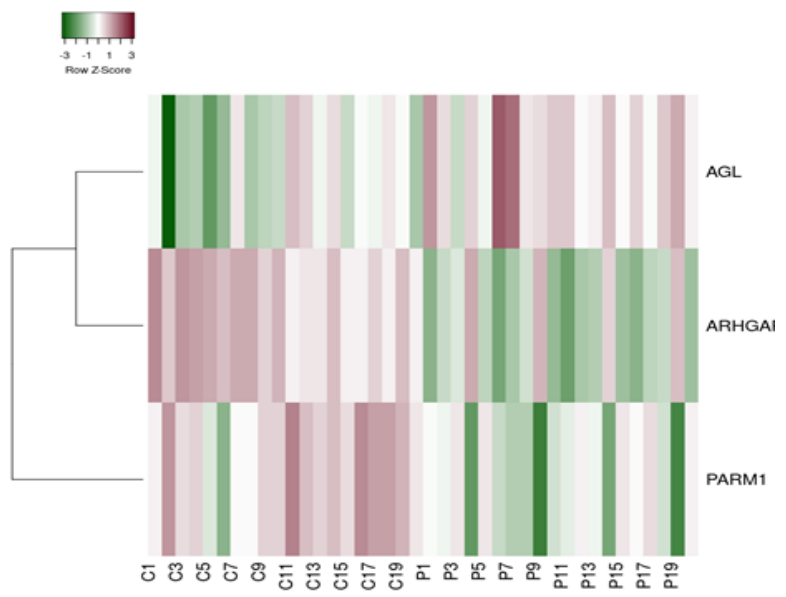

Fig. 13. Heatmap for $C M L$ biomarker identification in leukemia. There are many studies that have been reported (Prada et al., 2017, Jiang et al., 2016, Sweet et al., 2013) for the identification and characterization of biomarkers for individual classes of leukemia, but the present study was carried out for the comparative analysis of biomarker profiling of major classes (AML, ALL, CML, and CLL) of leukemia.

In the present study, the CD38 gene is a common differentially expressed gene in AML, ALL, and CLL. AUC analysis of CD38 shows, it is a good classifier marker for ALL and CLL. Jiang et al., (2016) also reported CD38 expression in HSCs, Leukemic stem cells, and B -ALL.

There are six differentially expressed genes that are commonly involved in acute leukemias. Out of these TSC22D3 and TNFRSF25 were selected on the basis of the lowest p-value. AUC analysis of TNFRSF25 shows that, it is a good classifier marker for acute leukemia. Miller et al., (2007) reported TSC22D3 (TSC22 domain family member) was highly induced in all the leukemic cells tested, regardless of apoptotic sensitivity

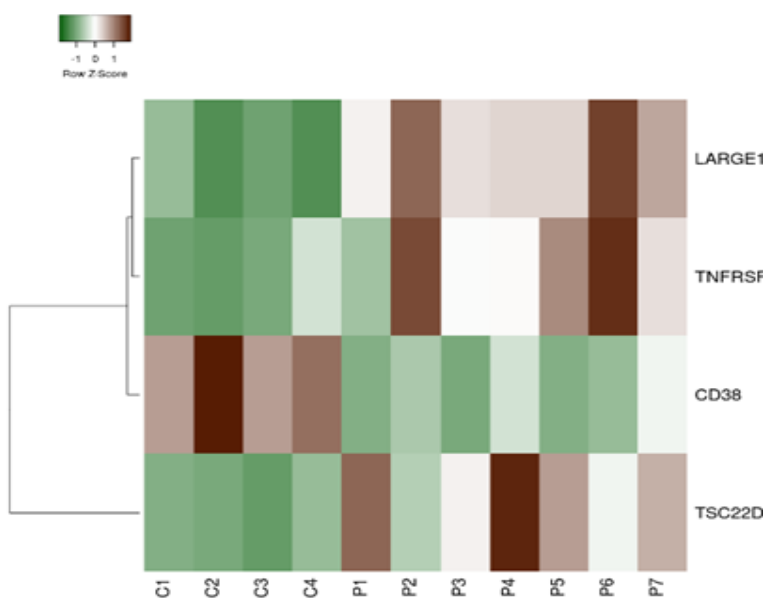

Fig. 12 Heatmap for ALL.

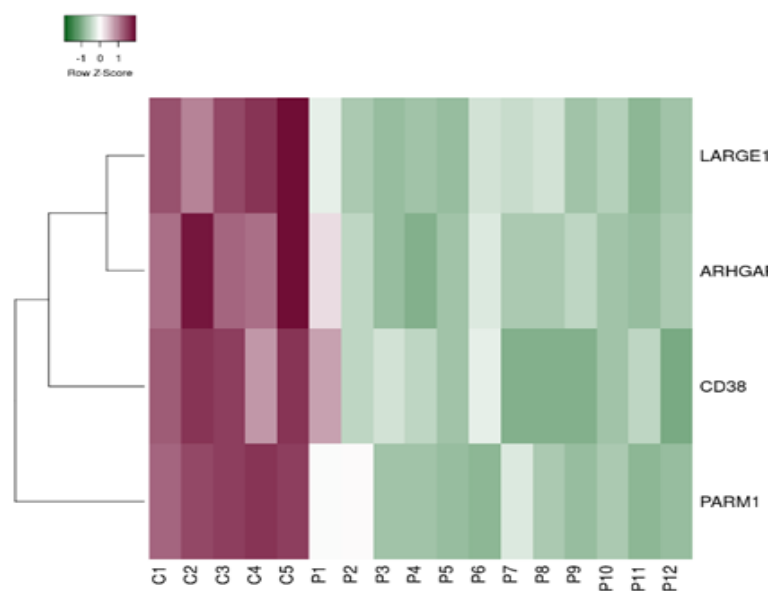

Fig. 14. Heatmap for CLL. 
or resistance. Yang et al. (2019) reported the role of upregulated TSC22D3 and stress-induced glucocorticoid surge in therapy-induced anticancer immunosurveillance. Paul et al., (2008) studied that TNFRSF25 (Tumor necrosis factor receptor superfamily member 25) are hypermethylated in tumor tissue. TNFRSF25 was significantly up-regulated in ECFCs (Endothelial colony-forming cells) from VTE (Venous thromboembolism) patients. Through experiments of functional validation, they further demonstrated that the upregulation of TNFSF15-TNFRSF25 axis sustains reduced survival and proliferation of ECFCs in VTE patients, thus suggesting that this molecular pathway, by impairing endothelial repair, may contribute to VTE pathogenesis.

Five genes were differentially expressed in chronic leukemia. Out of these ARHGAP32 and PARM1 were selected on the basis of the lowest p-value. AUC analysis of these two genes shows both are good classifier markers for CLL. A similar observation was reported by Vlaanderen et al. (2017), in which they reported ARHGAP32 as a pre-diagnostic blood transcriptome marker of chronic lymphocytic (CLL). Paulisally et al., (2014) identified PAMR1 (Peptidase domain-containing associated with muscle regeneration 1) as being frequently suppressed in breast cancer tissues. They reported that PAMR1 expression was reduced in all tested breast cancer cell lines, while PAMR1 was expressed moderately in normal breast tissues and primary mammary epithelial cells. Li (2020), reported the PARM1 key target gene for mir-382-5P regulating ovarian cancer.

In the present study, four common genes are differentially expressed in myeloid leukemia and six genes in lymphoid leukemia. AUC analysis of these genes showed AGL and TMEM154 is good classifier marker for AML and LARG1 is good classifier marker for both lymphoid leukemia. In the present study, the overall expression of AML (Fig. 2 and 3 Volcano plot and mean difference plot) showed that more differentially expressed genes were overexpressed. 164 genes were exclusively expressed in AML. Heatmap of CD38 showed that it is an overexpressed gene in AML and it is downregulated in ALL and CLL. In addition to CD38, AGL and TMEM154 are good classifier marker for AML. Crushell et al., (2010) confirmed that AGL encodes the glycogen debrancher enzyme, which is involved in glycogen degradation, and it was significantly altered, carbohydrate metabolic process in response to glucocorticoid stimulus. Guin et al., (2016) confirmed that AGL also involved in the induction of hyaluronic acid synthesis and further regulate tumor growth in bladder cancer. Holroyde et al., (1984) showed that diverse abnormalities of the carbohydrate metabolic process often occurred in cancer cachexia, such as colorectal cancer. In addition, aerobic glycolysis was ensured to be a metabolic adaptation that promotes the proliferation of colorectal cancer cells (Straus et al., 2013).

In the present study, the overall expression of ALL (Fig. 4 and 5 Volcano plot and mean difference plot) showed that more differentially expressed genes were underexpressed. 202 genes were exclusively expressed in ALL. In contrast with AML, the heatmap of CD38 showed that CD38 was an under-expressed gene in ALL. AUC analysis showed ARHGAP32, PARM1, and LARGE1 can be used as biomarkers for ALL. The overall expression of CLL and CML (Fig. 6- 9 Volcano plot and mean difference plot) showed that more differentially expressed genes were overexpressed. 146 genes were exclusively expressed in CLL and 194 genes were exclusively expressed in CML.

\section{Conclusion}

Using multiple bioinformatics tools and on the basis of the lowest adjusted $p$ value and AUC analysis present study proposed seven differentially expressed genes (CD38, TSC22D3, TNFRSF25, AGL, LARGE1, ARHGAP32, and PARM1) as biomarkers for leukemia. In conclusion, this study supports the accuracy of some formerly proposed biomarkers (CD38, and AGL) for leukemia, and also has suggested new candidate biomarkers (TSC22D3, TNFRSF25, LARGE1, ARHGAP32, and PARM1) which can be used as diagnostic or prognostic means or as therapeutic targets.

\section{Conflict of interest}

The authors declare that they have no conflict of interest.

\section{REFERENCES}

1. Aviles, S., Chavez, A., Hidalgo, A. \& Moreno, D. (2017). Global gene expression profiles of hematopoietic stem and progenitor cells from patients with chronic myeloid leukemia: the effect of in vitro culture with or without imatinib. Cancer Med, 6(12), 2942-2956.

2. Conesa, A., Madrigal, P., \& Tarazona, S. (2016). A survey of best practices for RNA-seq data analysis. Genome Biology, (17) 13.

3. Crushell, E., Treacy, E., Dawe, J., Durkie, M., \& Beauchamp, N. (2010). Glycogen storage disease type III in the Irish population. J Inherit Metab Dis., 33, S215-S218.

4. Gentleman, R.C., Carey, V.J., Bates, D.M., Bolstad, B., Dettling, M., Dudoit, S., Ellis, B., Gautier, L., Ge, Y., Gentry, J., Hornik, K., Hothorn, T., Huber, W., lacus, S., Irizarry, R., Leisch, F., Li, C., Maechler, M., Rossini, A.J., Sawitzki, G., Smith, C., Smyth, G., Tierney, L., Yang, J.Y., \& Zhang, J. (2004). Bioconductor: open software development for computational biology and bioinformatics. Genome Biology, 5(10), 80.

5. Guin, S., Ru, Y., Agarwal, N., Lew, C.R., Owens, C., Comi, G.P., \& Theodorescu, D. (2016). Loss of glycogen debranching enzyme AGL drives bladder tumor growth 
via induction of hyaluronic acid synthesis. Clin. Cancer Res., 1, 1274-1283.

6. Harder, L., Eschenburg, G., Zech, A., \& Kriebitzsch, N. (2013). Aberrant ZNF423 impedes B cell differentiation and is linked to adverse outcome of ETV6-RUNX1 negative B precursor acute lymphoblastic leukemia. J Exp Med., 21, 210(11), 2289-2304.

7. Heberle, H., Meirelles, G.V., \& Da Silva, FR. (2015). InteractiVenn: a web-based tool for the analysis of sets through Venn diagrams. BMC Bioinformatics, 16, 169. doi:10.1186/s12859-015-0611-3

8. Holroyde, C., Skutches, C., Boden, G., \& Reichard, G. (1984). Glucose metabolism in cachectic patients with colorectal cancer. Cancer Res., 44, 5910-5913.

9. Jiang, Z., Wu, D., \& Lin, S. (2016) CD34 and CD38 are prognostic biomarkers for acute $\mathrm{B}$ lymphoblastic leukemia. Biomark Res (4),23. doi: 10.1186/s40364-016-00805

10. Li, K., Wang, F., Cao, W.B., \& Lv, X.X. (2017). TRIB3 Promotes APL Progression through Stabilization of the Oncoprotein PML-RARa and Inhibition of p53-Mediated Senescence. Cancer Cell, 8, 31(5), 697-710.

11. Li, L., \& Wu, P. (2020). Analysis of the expression and clinical significance of miR-382-5P in ovarian cancer based on biological information. Research square, doi: 10.21203/rs.3.rs-16753/v1.

12. Miller, A.L., Komak, S., Webb, M.S., Leiter, E.H., \& Thompson, E.B. (2007). Gene expression profiling of leukemic cells and primary thymocytes predicts a signature for apoptotic sensitivity to glucocorticoids. Cancer Cell International, 7(1), 18. doi:10.1186/1475-2867-7-18.

13. Paul, A., Galler, J., Koss, M., Hagen, J., Turla, S., \& Campan, M. (2008). Identification of a panel of sensitive and specific DNA methylation markers for squamous cell lung cancer. Molecular Cancer, 7(1):62. doi: 10.1186/1476-4598-7-62.

14. Paulisally, L., Chizu, T., Toyomasa, K., Yusuke, N., \& Koichi, M. (2014). Identification of novel epigenetically inactivated gene PAMR1 in breast carcinoma. Oncology Reports, 33(1), 267-273. doi: 10.3892/or.2014.3581.

15. Prada, A. J., Arroyave, J.C., \& Röthlisberger, S. (2017) Molecular biomarkers in acute myeloid leukemia. Blood Rev. (1),63-76. doi: 10.1016/j.blre.2016.08.005

16. Rodriguez, E., \& Jiang, X. (2017). Differential gene expression in disease: a comparison between highthroughput studies and the literature. BMC Medical Genomics vol, 10, 59.

17. Sanchez, R., \& Mackenzie, S. A. (2020). Integrative
Network Analysis of Differentially Methylated and Expressed Genes for Biomarker Identification in Leukemia. Sci Rep., 10, 2123. doi:10.1038/s41598-020-581232.

18. Smyth, G.K. (2004). Linear models and empirical bayes methods for assessing differential expression in microarray experiments. Statistical applications in genetics and molecular biology, $3,3$.

19. Straus, D.S. (2013). TNFa and IL-17 cooperatively stimulate glucose metabolism and growth factor production in human colorectal cancer cells. Molecular Cancer, 12(1), 78. doi:10.1186/1476-4598-12-78

20. Sweet, K., Zhang, L. \& Pinilla-Ibarz, J. (2013) Biomarkers for determining the prognosis in chronic myelogenous leukemia. J Hematol Oncol (6), 54. doi:10.1186/17568722-6-54

21. Tang, Y., Zheng, J., Fu, X., \& Chen, Y. (2019). Bioinformatics Analysis of Differentially Expressed Genes and Their Functional Enrichment in Acute Myeloid Leukemia Bearing MLL Translocation. Biomark J., 5(2), 1.

22. Tripathi, S., \& Pandya, H. (2016). Discovery of Novel Gene Biomarker for Acute Myeloid Leukemia Through Differential Gene Expression Analysis. Annals of Applied Bio-Sciences, 3(1), e2349-6991.

23. Vargova, K., Curik, N., Burda, P., \& Basova, P. (2011). MYB transcriptionally regulates the miR-155 host gene in chronic lymphocytic leukemia. Blood, 117(14), 38163825.

24. Vlaanderen, J., Leenders, M., Hyam, M., Portengen, L., Kyrtopoulos, S., Berdahal I., Johanson, A., Hebels, D., Kok, T., Vineis, P., \& Vermeulen, R. (2017). Exploring the nature of prediagnostic blood transcriptome markers of chronic lymphocytic leukemia by assessing their overlap with the transcriptome at the clinical stage, $B M C \mathrm{Ge}$ nomics, 18, 239.

25. Wei, Fu., Cheng, G., Ding, Y., Deng, Y., \& Guo, P. (2020). Identification of hub genes and its correlation with the prognosis of acute myeloid leukemia based on high $\square$ throughput data analysis. Precision Radiation Oncology, 4(2), 49-56. doi: 10.1002/pro6.1089.

26. Yang, H., Xia, L., Chen, J., Zhang, S., Martin, V., Li, Q., Lin, S., Chen, J., Calmette, J., Lu, M., Fu, L., Lu, M., Fu, L., Yang, J., Pan, Z., Yu, K., He, J., Morand, E., Louf, G., Krzysiek, R., Zitvogel, L., Kang, B., Zhang, Z., Leader, A., Zhou, P., Lnfumev, L., Shi, M., Kroemer, G., \& Ma, Y. (2019). Stress-glucocorticoid-TSC22D3 axis compromises therapy-induced antitumor immunity. Nat Med., 25 (9), 1428-1441. doi: 10.1038/s41591-019-0566-4. 